Research Article

\title{
Evolutionary Game Analysis of Cross-Regional Coordinated Governance of Major Public Health Emergencies: The Example of the Spread of the COVID-19 Outbreak
}

\author{
Yao Xiao $\mathbb{D}^{1,2,3}$ Qiao Peng $\mathbb{C}^{2,3}$ and Wanting $X u \mathbb{C}^{1,3}$ \\ ${ }^{1}$ Center for Innovation and Development Studies, Beijing Normal University, Zhuhai 519000, China \\ ${ }^{2}$ Beijing Key Lab of Study on Sci-Tech Strategy for Urban Green Development, Beijing Normal University, Beijing 100875, China \\ ${ }^{3}$ Economics and Resource Management, Beijing Normal University, Beijing 100875, China
}

Correspondence should be addressed to Qiao Peng; 201731410009@mail.bnu.edu.cn and Wanting Xu; 202031410004@mail.bnu.edu.cn

Received 23 March 2021; Accepted 7 May 2021; Published 20 May 2021

Academic Editor: Luigi Rodino

Copyright (c) 2021 Yao Xiao et al. This is an open access article distributed under the Creative Commons Attribution License, which permits unrestricted use, distribution, and reproduction in any medium, provided the original work is properly cited.

\begin{abstract}
Cross-regional governance of government often faces various problems, which often brings great loss to the society. The global outbreak of the novel coronavirus pneumonia (NCP) in early 2020 has not only caused serious economic and social losses to various countries but also put the current public health event governance system to a severe test. The cross-regional character and spillover effects of public health outbreak governance often make it difficult to coordinate cross-regional governance. In this context, this paper adopts a regional evolutionary game analysis framework and studies the cross-regional governance of public health emergencies by constructing a symmetric game of peripheral regions and an asymmetric game of core-peripheral regions. The marginal contribution of this paper is to attempt to construct a symmetric game model for peripheral regions and an asymmetric game model for core and peripheral regions using an evolutionary game approach to study the behavioral strategies of multiple regions in the governance of public health emergencies, and it is found that when the regional spillover effects and governance costs are small or the economic and social damages caused by public health emergencies are large, all regions will choose to conduct coordinated governance. Otherwise, there will be regions that choose to "free-ride." This "free-rider" mentality has led to the failure in achieving good cross-regional collaborative governance of public health emergencies, resulting in a lack of efficiency in the overall governance of public health in society. However, when the spillover effect of regional governance exceeds a certain critical value, the result of the regional governance game is also the socially optimal result, when public health emergencies are effectively governed. At the same time, the relevant findings and analytical framework of this paper will provide a policy reference for the cross-regional governance of the current new crown epidemic.
\end{abstract}

\section{Introduction}

The outbreak of the novel coronavirus (COVID-19) in early 2020 caused a huge impact on the social and economic stability in a short period, with countries experiencing economic shutdowns to a certain extent, causing incalculable losses to economies and societies around the world, as well as posing a serious challenge to the emergency management systems and capabilities of countries with public health emergencies. The initial period coincided with the early stages of the Chinese Traditional Spring Festival, and with the large-scale population movement, the epidemic spread rapidly to the surrounding provinces and the whole country, with Wuhan being the center. Among them, Henan, Hunan, Anhui, Jiangxi, Guangdong, Jiangsu, Chongqing, Sichuan, Shandong, and Zhejiang were the main destinations of outflows and also became the provinces with the most serious outbreaks in China, except for Hubei [1]. The massive population movement has gradually turned the new crown epidemic into a major public health event with global repercussions, causing significant economic losses and human casualties [2]. The shortcomings of the current 
public health emergency governance model in response to major public health emergencies have been exposed [3].

Public health events are public measures taken by the public sector to prevent diseases, extend life expectancy, and improve health. These include not only public health events caused by nondiseases but also those caused by epidemic diseases and so on $[4,5]$. In recent years, repeated outbreaks of major public health events have not only been severe and harmful but also often have a cross-regional character $[6,7]$, and their management needs to rely on cooperation among multiple regions. China has made it clear since 2018 that public health events should be governed by a model of coordinated governance of multiple subjects and multiple regions with a common interest as the goal. However, due to the differences in interests between regions, the externalities of regional governance, and the uncertainty and complexity of regional governance decisions, it is difficult to achieve coordinated governance across regions. The difficulty is mainly reflected in the following ways: (1) The association and conflict of interests of multiregional subjects exacerbate the complexity of their governance decisions, thus making it more difficult to coordinate multiregional governance behaviors. The governance of public health emergencies has a significant regional spillover effect, and local government decisions have an impact on the interests and decisions of other regions, while the governance of public health emergencies often requires the suppression of economic activities at the cost of the incentive to "free-ride," which leads many regions to choose nongovernance or lax governance. They can enjoy the benefits of governance in other regions while reducing the impact and cost of governance or strict governance on the local economy. It reduces the efficiency of governance of public health emergencies, making it difficult to effectively control public health emergencies and causing greater losses to the society and economy. (2) The uncertainty of multiregional decision-making leads to the lack of stability of multiregional collaborative governance of public health emergencies. When each region pursues the maximization of its own interests, the mutual influence of interregional interests makes the region's governance decisions inevitably dependent on other regional policies. Considering the asymmetry of information related to interregional decision-making and the severity of public health emergencies, regions will inevitably have to constantly adjust their strategies based on the relevant information they receive, thus increasing the uncertainty of regional governance decisions and adding difficulties to the governance of public health emergencies. Therefore, there is a need to provide a framework for analyzing the decisionmaking behavior of multistakeholders in a complex and uncertain environment, to provide a reference for future government policy formulation in the face of cross-regional governance of major public safety emergencies.

Evolutionary game (EGM) is a new development in game theory in recent years, which originally arose in the field of biology and was first proposed by Smith et al. [8] in their study of symmetric population games. They also clarified the basic concept of the evolutionary game-evolutionarily stable strategy (ESS). Their development abandoned the assumption of perfect rationality in classical game theory and replaced it with the assumption of bounded rationality, combining the equilibrium analysis in traditional game theory with the dynamic, evolutionary analysis in biology, thus giving rise to evolutionary game theory [9]. In the 1990s, economists and social scientists used biological evolutionary game theory to study socioeconomic problems and made breakthroughs especially in the areas of transition and formation of social systems, social governance, and so forth [10]. The evolutionary game assumes that the participants are not rational individuals with infinite reasoning ability and cannot precisely calculate the Nash equilibrium strategies and make corresponding choices like the individuals in traditional games, but continuously learn and adjust their strategies and gradually converge to a stable Nash equilibrium strategy according to the results of each game in the process of repeated games. Therefore, by constructing a multiregional evolutionary game model on the governance of public health emergencies, we can well capture the complexity and uncertainty in the process of public health emergencies governance and provide a good analytical framework for the study of cross-regional coordinated governance of public health emergencies.

Currently, less attention has been paid to the regional governance of public health emergencies in existing studies. The studies mainly focus on proposing suggestions for improving the construction of public health governance systems using case studies [11-14]. Some scholars have also used traditional statistical regression methods and big data statistical methods to study the factors for the governance efficiency of public health events [15-18] to achieve accurate governance and prevention and control of major public health emergencies. Research on the governance of public health emergencies using game theory has mainly focused on the government, social organizations, and the public, while research on how to effectively achieve multiregional cooperation in governance is scarce. Yang et al. [19] used evolutionary game tools to study the governance decisions between the government and health organizations in the governance of public health emergencies. Le et al. [20] and Liu et al. [21] used evolutionary games to study the diffusion mechanism of epidemics and how the government and the public participate in the comanagement of epidemics. A few other scholars [22, 23] studied the influence of media on the governance of public health emergencies by constructing a game model of the prevention and control center, media, and the public.

In summary, existing studies hardly cover the issue of multiregional collaborative governance in public health emergencies, and especially the literature using the evolutionary game framework is even scarcer. To this end, the marginal contribution of this paper is to attempt to construct a symmetric game model for peripheral regions and an asymmetric game model for core and peripheral regions using an evolutionary game approach to study the behavioral strategies of multiple regions in the governance of public health emergencies. On this basis, the equilibrium state and governance efficiency of the cross-regional governance game of major public health events are analyzed and relevant policy recommendations are proposed as a reference for the current new crown epidemic as well as the 
multiregional coordinated governance decisions of possible sudden major public events in the future.

\section{Governance Game for Public Health Emergencies in Peripheral Regions}

It is assumed that the core area is where the outbreak of a major public health event takes place, and the area around the core area is the peripheral area. Another assumption is that the core area is faced with a more serious situation that the public health event can spread to the peripheral area. In this section, an evolutionary game model is constructed for the collaborative governance of peripheral regions to analyze the collaborative governance of public health emergencies among peripheral regions, and a game model is constructed for the core region and peripheral regions in Section 3 to analyze the collaborative governance of public health emergencies between the core region and peripheral regions.

2.1. Model Construction. Suppose there are two symmetric peripheral regions around the core region where a public health event occurs, facing a possible public health outbreak in their respective jurisdictions, such as being at risk of a large-scale spread of the new coronavirus virus, which will cause great damage to the economy, society, and lives in case of widespread spread. The loss is denoted by $\pi$. The governments of the peripheral regions will manage the public health event by curbing economic activity, using social resources, etc., incurring a governance cost of $C$. Because of the extremely destructive nature of public health emergencies, it is assumed that the cost of a public event outbreak is much greater than the cost of governance, that is, $\pi>>C$. When only one peripheral region is engaged in the governance, the probability of an outbreak in that region is $p$ and there is a spillover effect to the other peripheral region. The larger the spillover effect, the lower the probability of an outbreak in the region with no governance, with zero cost of governance. Assume that the size of the spillover effect of governance in one peripheral region on another peripheral region is $\alpha p$, which denotes the probability of a public health outbreak in the region with no governance, where $\alpha(1 \leq \alpha \leq(1 / p))$ is the spillover coefficient, and when $\alpha=1$, the probability of an outbreak in the nongovernance region will depend entirely on the region with governance. In other words, the region with no governance can fully enjoy the externality generated by the region with governance. When the spillover effect generated by the governance region is zero $(\alpha=0)$, the nongovernance region cannot enjoy the externality from the governance region at all, at which point the region without any governance is bound to encounter a public health outbreak. Then, the probability is 1 . Therefore, a larger $\alpha$ means a smaller spillover effect and fewer benefits to the free-riding nongovernance region, and vice versa. Meanwhile, only when both regions take part in joint governance can the public health events in the two regions be completely controlled, with a probability of an outbreak at 0 . Therefore, based on the above assumptions, the payoff matrix of the public health event governance game in the periphery is shown in Table 1.

This paper further assumes that decision-makers in all regions are boundedly rational, and in the process of the regional governance game on public health events, their decisions are constantly adjusted to maximize the benefits of their regions based on the information obtained, and therefore, their decisions are often uncertain and constantly changing with the governance strategies of the other region. Therefore, it is assumed that peripheral region 1 chooses its governance strategy with a certain probability $x$ and constantly adjusts its probability to maximize the benefit of its region. Similarly, it is assumed that peripheral region 2 chooses its governance strategy with a certain probability $y$ and constantly adjusts its probability to maximize its benefit. The strategy adjustment follows the replicator dynamic equation with the following expression [24-26]:

$$
F\left(x_{v}\right)=\frac{\mathrm{d} x_{v}}{\mathrm{~d} t}=x_{v}\left[E\left(x_{v}\right)-\bar{E}\right]
$$

where $x_{v}$ denotes the probability that a participant in the game will use a strategy $v, E\left(x_{v}\right)$ denotes the expected payoff for the participant using the strategy $v$, and $\bar{E}$ denotes the average payoff for a participant choosing all possible strategies.

2.2. Solving Game Equilibrium. Based on the payoff matrix of the peripheral region game and the replicator dynamics equation, the replicator dynamics equation for the strategy adjustment in peripheral region 1 and peripheral region 2 can be derived.

(1) Let $U_{1,0}$ denote the expected return obtained when peripheral region 1 adopts a governance strategy, $U_{1,1}$ the expected return obtained when peripheral region 1 adopts nongovernance strategy, and $U_{1}$ the average return obtained by peripheral region 1 .

Specifically,

$$
\begin{aligned}
U_{1,0} & =-C y+(-\pi p-C)(1-y), \\
U_{1,1} & =-\pi \alpha p y-\pi(1-y), \\
U_{1} & =x U_{1,0}+(1-x) U_{1,1} .
\end{aligned}
$$

Then, the replicator dynamics equation for the strategy adjustment in peripheral region 1 is as follows:

$$
\begin{aligned}
\frac{\mathrm{d} x}{\mathrm{~d} t} & =x\left(U_{1,0}-U_{1}\right) \\
& =x(1-x)\{[(1+\alpha) \pi p-\pi] y-(\pi p+C-\pi)\} .
\end{aligned}
$$


TABLE 1: Matrix of game gains in the peripheral regions.

\begin{tabular}{lcc}
\hline Peripheral region 1 & \multicolumn{2}{c}{ Peripheral region 2} \\
& Governance & Nongovernance \\
\hline Governance & $-C,-C$ & $-\pi p-C,-\pi \alpha p$ \\
Nongovernance & $-\pi \alpha p,-\pi p-C$ & $-\pi,-\pi$ \\
\hline
\end{tabular}

When the strategy adjustment in peripheral region 1 stabilizes, i.e., $(\mathrm{d} x / \mathrm{d} t)=0$,

$$
\begin{aligned}
& x_{1}^{*}=0, \\
& x_{2}^{*}=1, \\
& y_{3}^{*}=a=\frac{\pi p+C-\pi}{(1+\alpha) \pi p-\pi} .
\end{aligned}
$$

(2) Let $U_{2,0}$ denote the expected return obtained when peripheral region 2 adopts a governance strategy, $U_{2,1}$ the expected return obtained when peripheral region 2 adopts a nongovernance strategy, and $U_{2}$ the average return obtained by peripheral region 2 .

Similarly, the replicator dynamics equation for strategy adjustment in peripheral region 2 can be obtained as follows:

$$
\begin{aligned}
\frac{\mathrm{d} y}{\mathrm{~d} t} & =y\left(U_{2,0}-U_{2}\right) \\
& =y(1-y)\{[(1+\alpha) \pi p-\pi] x-(\pi p+C-\pi)\} .
\end{aligned}
$$

When the strategy adjustment in peripheral region 2 stabilizes, i.e., $(\mathrm{d} y / \mathrm{d} t)=0$,

$$
\begin{aligned}
& y_{1}^{*}=0, \\
& y_{2}^{*}=1, \\
& x_{3}^{*}=b=\frac{\pi p+C-\pi}{(1+\alpha) \pi p-\pi} .
\end{aligned}
$$

When $(\mathrm{d} x / \mathrm{d} t)=0$ and $(\mathrm{d} y / \mathrm{d} t)=0$, there are four equilibrium points in the evolutionary game about the governance of public health events in the peripheral region: $(0,0),(0,1),(1,0),(1,1)$, and $(b, a)$.

2.3. Stability Analysis of Equilibrium Solutions. Let $F(x)=(\mathrm{d} x / \mathrm{d} t), G(y)=(\mathrm{d} y / \mathrm{d} t)$ analyze the stability of each local equilibrium point of this two-dimensional dynamical system using the Jacobian matrix:

$$
\begin{aligned}
J & =\left[\begin{array}{cc}
\frac{\partial F(x)}{\partial x} & \frac{\partial F(x)}{\partial y} \\
\frac{\partial G(y)}{\partial x} & \frac{\partial G(y)}{\partial y}
\end{array}\right]=\left[\begin{array}{ll}
c_{11} & c_{12} \\
c_{21} & c_{22}
\end{array}\right], \\
c_{11} & =(1-2 x)\{[(1+\alpha) \pi p-\pi] y-(\pi p+C-\pi)\}, \\
c_{12} & =x(1-x)[(1+\alpha) \pi p-\pi], \\
c_{21} & =y(1-y)[(1+\alpha) \pi p-\pi], \\
c_{22} & =(1-2 y)\{[(1+\alpha) \pi p-\pi] x-(\pi p+C-\pi)\} .
\end{aligned}
$$

The stable equilibrium point of the replicator dynamic equation satisfies the following two conditions:

$$
\begin{aligned}
\operatorname{tr}(J) & =c_{11}+c_{22}<0, \\
\operatorname{det}(J) & =c_{11} c_{22}-c_{12} c_{21}>0 .
\end{aligned}
$$

From the Jacobian matrix of the replicator dynamic equation, Table 2 is obtained.

According to the stability condition for the equilibrium solution of the evolutionary game, the following proposition can be derived.

Proposition 1. When $\alpha>(C / \pi p)$, there exists a unique stable equilibrium point $(1,1)$ for the evolutionary game of public health event governance in the peripheral region; that is, the peripheral regions eventually choose (governance, governance) and remain stable.

When the externalities of governance in one peripheral region are too small for other peripheral regions, both sides would choose to govern, and the benefits of "free-riding" in the nongovernance region are too small to significantly reduce the probability of public health outbreaks in the region, resulting in insufficient expected benefits for the peripheral regions in taking the decision not to govern. Thus, the choice to govern is mutually beneficial. At the same time, when the spillover effect is certain, the smaller the cost of governance and the greater the harm caused by a public health outbreak to the regional economy and society, both regions tend to choose governance, and from the perspective of economic efficiency, the outcome of the game is socially 
TABLE 2: Analysis of local equilibrium points.

\begin{tabular}{lcccc}
\hline Equilibrium points & $c_{11}$ & $c_{12}$ & $c_{21}$ & $c_{22}$ \\
\hline$(0,0)$ & $-(\pi p+C-\pi)$ & 0 & 0 & $-(\pi p+C-\pi)$ \\
$(0,1)$ & $\alpha \pi p-C$ & 0 & 0 & $\pi p+C-\pi$ \\
$(1,0)$ & $\pi p+C-\pi$ & 0 & 0 & $\alpha \pi p-C$ \\
$(1,1)$ & $-(\alpha \pi p-C)$ & 0 & 0 & $-(\alpha \pi p-C)$ \\
$(a, b)$ & 0 & $c_{12}^{*}$ & $c_{21}^{*}$ & 0 \\
\hline
\end{tabular}

optimal. Therefore, public health events can be effectively controlled with the collaboration of multiple regions. For the current new crown epidemic, some peripheral regions believe that once the epidemic breaks out locally, it will cause great damage to the local economy and society. Additionally, even if other peripheral regions choose relevant governance measures, such as city closure and production shutdown, the positive spillover effect on the region is small, so many peripheral regions actively take relevant measures to suppress the spread and outbreak of the epidemic in their own regions. Thus, an evolutionary game equilibrium with joint governance of all peripheral regions is formed.

Proposition 2. When $\alpha<(C / \pi p)$, there are two stable equilibria $(0,1)$ or $(1,0)$ in the evolutionary game between the peripheral regions; i.e., they eventually choose (governance, nongovernance) or (nongovernance, governance), which then remain unchanged. The phase diagram of its evolutionary game is shown in Figure 1, where the coordinates of point $H$ are $(b, a)$, and since the game for the peripheral regions is symmetric, i.e., $a=b$, the probability of convergence to points $A$ and $C$ is the same, i.e., $P_{A}=P_{C}=(1 / 2)$.

When the externalities of the governance area on the nongovernance area are large enough and exceed a certain threshold, one party chooses to govern and the other chooses to "free-ride" on the benefits of the area with governance, both seek to control the risk of public health outbreaks in the region through the spillover effects and to save on the cost of governance. At this point, the benefits are higher for the party with no governance than for the governance party. At the same time, the greater the cost of governance, the less economic harm caused by public health events, so there is always a tendency for one party to choose to "free-ride." At this point, multiregional cooperation in the management of public health events is not possible. In the case of the current new crown epidemic, for example, some peripheral regions are considering whether to adopt governance or control measures such as city closure or suppression of economic activities, but these measures will cause local economic losses and affect local economic development and people's normal life. As a result, some peripheral regions adopted stringent governance measures while others adopted simple response measures, either by not adopting relevant governance measures or by taking relatively lenient governance measures while enjoying the spillover effects of strict governance of the new epidemic in other regions, thus maximizing the benefits to the region.

However, even if regional cooperation in governance cannot be achieved, an equilibrium outcome where only one

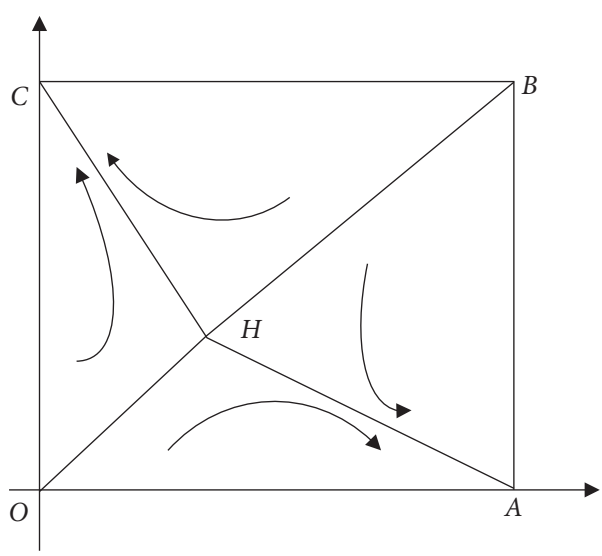

FIgURe 1: Phase diagram of game evolution with peripheral regions.

party governs is not necessarily inefficient. The details are as follows.

Proposition 3. When $(C / \pi p)-1<\alpha<(C / \pi p)$, the equilibrium state in which the game of public health event governance in the peripheral regions finally converges to (governance, nongovernance) or (nongovernance, governance) is not socially optimal. The optimal state is one in which both sides govern, so the public health event is not effectively governed in this case.

The proof is as follows: when the peripheral regions all choose to govern, the total social benefit is $U_{S, 1}=-2 C$; when the peripheral regions choose (governance, nongovernance) or (nongovernance, governance), the total social benefit is $U_{S, 2}=-(1+\alpha) \pi p-C$. Since $(C / \pi p)-1<\alpha<(C / \pi p), U_{S, 2}$ $<U_{S, 1}$. In this case, the equilibrium is not socially optimal at this time, and "free-ride" for the nongovernance region will lead to insufficient social governance for public health events, which cannot be effectively controlled.

Proposition 4. When $\alpha<(C / \pi p)-1$, then $U_{S, 2}>U_{S, 1}$. Specifically, the stable equilibrium of the game (governance, nongovernance) or (nongovernance, governance) is socially optimal, and the equilibrium outcome of the game corresponds to the socially optimal outcome. This is because when the spillover effect from the governance region is large enough to allow the other region to control local public health events without governance, there is no need for all regions to be governed and to waste resources on governance. As such, the regional game results in a socially optimal outcome with public health events can be effectively controlled.

Therefore, according to Propositions 3 and 4, for the current management of the new crown epidemic, although some peripheral regions have the speculative mentality to "free-ride" on the governance of other regions, resulting in no joint regional governance in the evolutionary game in the peripheral regions, as long as the spillover from regional 
governance to other regions is large enough, it is best for some regions to choose not to govern.

\section{The Core-Periphery Regional Public Health Emergencies Governance Game}

This section focuses on the analysis of the evolutionary game model between the core area where a public health emergency occurs and its peripheral area and analyzes the issue of synergistic management of public health emergencies in the core and peripheral regions.

3.1. Model Construction. This section still assumes the existence of two regions, a core region 1 where a public health event breaks out and a peripheral region 2 surrounding that core region. The core region is the origin of the public health event, with a higher probability of a large-scale spread and greater difficulties in governance. Thus, it is assumed that the governance cost of core region 1 is greater than that of peripheral region 2 , i.e., $C_{1}>C_{2}$. When the core region is governed but the peripheral region is not, the governance of the core region will exert a spillover effect on the peripheral region. The size of the spillover is $\beta q$, where $q$ denotes the probability of a public health outbreak in the region when only one region is governed, and the spillover coefficient $1<\beta<(1 / q)$ that measures the spillover effect of the core region to the peripheral regions. It is further assumed that there is no spillover effect of governance in the peripheral region to the core region because public health outbreaks in the core region are more severe, governance in the peripheral region simply cannot have an impact on the core region, and the peripheral region cannot control local public health events alone and will be affected by the spread of public health events in the core region at any time and then suffer from the outbreak again. Therefore, as long as the core region is not governed, the probability of public health outbreaks in the peripheral region is 1 , and the impact of governance in the peripheral region on the core region is almost 0 . When the two regions join forces to govern, public health events in all regions can be completely controlled, during which both regions pay the cost of governance. Based on the above assumptions, the payoff matrix of the public health event governance game between the core and peripheral regions is shown in Table 3.

It is assumed that core region 1 chooses a governance strategy with a certain probability $\mu$ and continuously adjusts the probability to maximize its benefits and that peripheral region 2 chooses a governance strategy with a certain probability $v$ and continuously adjusts the probability to maximize its benefits.

3.2. Solving Game Equilibrium. Based on the payoff matrix of the core-periphery region game and the replicator
TABLE 3: Core and periphery game benefit matrix.

\begin{tabular}{lcc}
\hline Core region 1 & \multicolumn{2}{c}{ Peripheral region 2 } \\
& Governance & Nongovernance \\
\hline Governance & $-C_{1},-C_{2}$ & $-\pi q-C_{1},-\pi \beta \mathrm{q}$ \\
Nongovernance & $-\pi,-\pi q-C_{2}$ & $-\pi,-\pi$ \\
\hline
\end{tabular}

dynamics equation, the replicator dynamics equation for the game strategy adjustment in core region 1 and periphery region 2 can be derived.

(1) Let $U_{c, 0}$ denote the expected return obtained when core area 1 adopts a governance strategy, $U_{c, 1}$ the expected return obtained when core area 1 adopts a nongovernance strategy, and $U_{c}$ the average return obtained by core area 1 .

Specifically,

$$
\begin{aligned}
U_{c, 0} & =-C_{1} v+\left(-\pi q-C_{1}\right)(1-v), \\
U_{c, 1} & =-\pi \\
U_{c} & =\mu U_{c, 0}+(1-\mu) U_{c, 1} .
\end{aligned}
$$

Then, the replicator dynamics equation for the core region 1 strategy adjustment is as follows:

$\frac{\mathrm{d} \mu}{\mathrm{d} t}=\mu\left(U_{c, 0}-U_{c}\right)=\mu(1-\mu)\left[\pi q v-\left(\pi q+C_{1}-\pi\right)\right]$.

When the core region 1 strategy adjustment stabilizes, i.e., $(\mathrm{d} \mu / \mathrm{d} t)=0$,

$$
\begin{aligned}
& \mu_{1}^{*}=0, \\
& \mu_{2}^{*}=1, \\
& v_{3}^{*}=\frac{\pi q+C_{1}-\pi}{\pi q} .
\end{aligned}
$$

(2) Let $U_{p, 0}$ denote the expected return obtained when peripheral region 2 adopts a governance strategy, $U_{p, 1}$ the expected return obtained when peripheral region 2 adopts a nongovernance strategy, and $U_{p}$ the average return obtained by peripheral region 2 .

Specifically,

$$
\begin{aligned}
U_{p, 0} & =-C_{2} \mu+\left(-\pi q-C_{2}\right)(1-\mu), \\
U_{p, 1} & =-\pi \beta q \mu-\pi(1-\mu), \\
U_{p} & =v U_{p, 0}+(1-v) U_{p, 1} .
\end{aligned}
$$


Then, the replicator dynamics equation for the strategy adjustment in peripheral region 2 is as follows:

$$
\begin{aligned}
\frac{\mathrm{d} v}{\mathrm{~d} t} & =v\left(U_{p, 0}-U_{p}\right) \\
& =v(1-v)\left\{[\pi \beta q-\pi(1-q)] \mu+\pi(1-q)-C_{2}\right\} .
\end{aligned}
$$

When the strategy adjustment in core region 1 stabilizes, $(\mathrm{d} \mu / \mathrm{d} t)=0$.

When $(\mathrm{d} \mu / \mathrm{d} t)=0$ and $(\mathrm{d} v / \mathrm{d} t)=0$, there are four equilibria in the core-periphery game on the governance of public health events: $(0,0),(0,1),(1,0),(1,1)$, and $\left(\mu_{3}^{*}, v_{3}^{*}\right)$.

3.3. Stability Analysis of Equilibrium Solutions. Similarly, let $F(\mu)=(\mathrm{d} \mu / \mathrm{d} t), G(v)=(\mathrm{d} v / \mathrm{d} t)$ analyze the stability of each local equilibrium point of this two-dimensional dynamical system using the Jacobian matrix:

$$
\begin{aligned}
& J=\left[\begin{array}{ll}
\frac{\partial F(\mu)}{\partial \mu} & \frac{\partial F(\mu)}{\partial v} \\
\frac{\partial G(v)}{\partial \mu} & \frac{\partial G(v)}{\partial v}
\end{array}\right]=\left[\begin{array}{ll}
c_{11} & c_{12} \\
c_{21} & c_{22}
\end{array}\right] \\
& c_{11}=(1-2 \mu)\left[\pi q v-\left(\pi q+C_{1}-\pi\right)\right], \\
& c_{12}=\mu(1-\mu) \pi q, \\
& c_{21}=v(1-v)[\pi \beta q-\pi(1-q)], \\
& c_{22}=(1-2 v)\left\{[\pi \beta q-\pi(1-q)] \mu+\pi(1-q)-C_{2}\right\} .
\end{aligned}
$$

Similarly, from the Jacobian matrix of the replicator dynamics equation for the core-periphery game, Table 4 is obtained.

The following proposition can be derived from the stable equilibrium conditions for evolutionary games.

Proposition 5. When $\beta>\left(C_{2} / \pi q\right),(1,1)$ of the evolutionary game between the core and peripheral regions is the only stable equilibrium, the core and peripheral regions in the public health event governance game finally choose (governance, governance) and remain stable. Besides, this equilibrium state is socially optimal. Therefore, when the spillover effect from governance in the core region and the cost of governance in the peripheral region are smaller, or when the harm by the public health event is greater, both the core region and the peripheral region tend to choose governance, and the state is Pareto optimal. For the current governance of the new crown epidemic, the core region of the outbreak chooses to govern due to the great harm the outbreak generates, but its governance has a small spillover effect on some peripheral regions. If the peripheral regions do not choose strict governance and control measures, there will be a greater risk of an outbreak, and the peripheral regions eventually have to cooperate with the core region to
TABLE 4: Analysis of local equilibrium points.

\begin{tabular}{lcccc}
\hline Equilibrium points & $c_{11}$ & $c_{12}$ & $c_{21}$ & $c_{22}$ \\
\hline$(0,0)$ & $-\left(\pi q+C_{1}-\pi\right)$ & 0 & 0 & $\pi(1-q)-C_{2}$ \\
$(0,1)$ & $-\left(C_{1}-\pi\right)$ & 0 & 0 & $-\left[\pi(1-q)-C_{2}\right]$ \\
$(1,0)$ & $\pi q+C_{1}-\pi$ & 0 & 0 & $\pi \beta q-C_{2}$ \\
$(1,1)$ & $C_{1}-\pi$ & 0 & 0 & $-\left(\pi \beta q-C_{2}\right)$ \\
$\left(\mu_{3}^{*}, v_{3}^{*}\right)$ & 0 & $c_{12}^{*}$ & $c_{21}^{*}$ & 0 \\
\hline
\end{tabular}

jointly govern the epidemic due to the trade-off between the cost of governance and the risk of outbreaks.

Proposition 6. When $\left(C_{2} / \pi q\right)-1<\beta<\left(C_{2} / \pi q\right),(1,0)$ is the only stable equilibrium point when the core region and the peripheral region finally choose (governance, nongovernance) and remain stable, the equilibrium state is not socially optimal. In other words, when the peripheral region believes that the governance of the core region can produce a sufficiently large spillover effect and the cost of governance is large, or when the harm caused by public health events is small, the speculative mentality of the peripheral region to "free-ride" leads to its choice of nongovernance, thus resulting in the core region and the peripheral region not being able to achieve joint governance, and public health emergencies cannot be effectively controlled. As a result, public health governance is inadequate for society as a whole. For the current management of the new crown epidemic in the core and peripheral regions, the surrounding areas of the core region have chosen to strictly control and manage the local new crown epidemic, and there are few cases of nonmanagement. This is mainly because the epidemic is different from ordinary influenza in terms of a higher risk of transmission, lower recovery rate, greater harm, and a smaller spillover effect from the management of the core region, so they all choose to work with the core region.

\section{Game Analysis of the Governance of the "Free- Rider" Phenomenon}

According to the above analysis, when public health governance in one region has a large spillover effect on other regions, the latter will choose to "free-ride" and adopt a nongovernance strategy. However, not all "free-rider" phenomena need to be governed. Only when the spillover effect of the governance region on other regions is within a certain range, the game outcome of regional governance is not socially optimal, and public health emergencies are not effectively governed. Therefore, this section investigates the management of the inefficient "free-rider" phenomenon through the establishment of a joint regional management platform, i.e., by setting up a joint cross-regional public health governance platform or management body to manage regional governance in an integrated manner and providing rewards and penalties to nongovernance areas, thereby offering incentives for joint regional governance. To illustrate the moderating effect of a cross-regional public health governance platform on regional governance, a symmetric game in peripheral areas is used as an example. Assuming 
TABLE 5: Game benefit matrix with the intervention of a crossregional public health governance platform.

\begin{tabular}{lcc}
\hline \multirow{2}{*}{ Peripheral region 1} & \multicolumn{2}{c}{ Peripheral region 2 } \\
& Governance & Nongovernance \\
\hline Governance & $-C,-C$ & $-\pi p-C,-\pi \alpha p-v$ \\
Nongovernance & $-\pi \alpha p-v,-\pi p-C$ & $-\pi-v,-\pi-v$ \\
\hline
\end{tabular}

that the cross-regional public health governance management platform penalizes the nongovernance regions with $v$, the symmetric game matrix is shown in Table 5 .

Based on the stability analysis of the equilibrium solution of the evolutionary game in the peripheral regions in Section 2.3 , we can infer that when the spillover effects from regional governance $\alpha$ fall into the $((C-v / \pi p)-1$, the $(C-v / \pi p))$ interval, the stable solution of the evolutionary game is either (governance, nongovernance) or (nongovernance, governance), and the equilibrium is not socially optimal. Moreover, the nongovernance region "free-rides" on the governance region, leading to insufficient social governance. Since the spillover coefficient $1<\alpha<(1 / p)$, so only when $(C-v / \pi p) \leq 1$, that is, when the cross-regional joint public health governance platform penalizes the nongovernance region $v \geq C-\pi p$, there is no intersection between the two sets that the spillover effect has to satisfy. In this case, the equilibrium of the regional governance game is socially optimal, and public health emergencies are effectively managed. At the same time, the conclusion also holds for the public health event governance game between the core region and the peripheral region, where there exists a critical threshold for government penalties that allow for the effective governance of public health emergencies. Therefore, for the current governance of the epidemic, to ensure effective multiregional joint governance, a multiregional joint management platform can be established for coordination and control, and effective multiregional collaborative governance can be achieved through rewards and penalties.

\section{Conclusions and Recommendations}

In the context of the spread of the "new coronavirus," achieving cross-regional coordinated governance of public health emergencies has become an important issue that needs to be addressed urgently. The complexity and dynamics of cross-regional governance of public health emergencies increase the difficulty of regional collaborative governance. This paper employs the method of an evolutionary game in biology and constructs the peripheral region evolutionary game model and core-peripheral region evolutionary game model, which captures the complexity and dynamic nature of the decision-making process of regional governance. Additionally, the models provide a good analytical framework for a systematic analysis of the cross-regional governance of public health emergencies. The findings of this study are as follows:

(1) In the game of governance of public health emergencies between peripheral regions, when the spillover effect of regional governance on nongovernance regions is small enough, that is, when the benefits of "free-riding" in nongovernance regions are inadequate, all peripheral regions tend to choose cooperative governance; when the cost of regional governance of public health emergencies is small and the economic and social harm caused by public health emergencies is greater, all regions tend to choose joint governance. Concurrently, when the externalities of governance in one region on nongovernance regions are large, some regions will choose to "free-ride" on the benefits of other governance regions for free. Thus, the peripheral regions cannot achieve joint governance, and public health emergencies cannot be effectively controlled. Unless the externalities generated by governance are large enough for governance in one region to control public health emergencies for all regions, partial regional governance cannot achieve effective management of the overall public health events. For the current governance of the new crown epidemic, some peripheral regions are considering whether to adopt relevant governance or control measures such as city closure or suppression of economic activities, but these measures will result in economic losses to the local area and affect the local economic development as well as the livelihood of the people. In this regard, some regions with an opportunistic mentality tend to take advantage of the "governance of other regions" through inaction or lenient approaches, while enjoying the spillover effect from the other regions to maximize their benefits. As a result, some peripheral regions have adopted strict control measures while others have taken simple measures to deal with the epidemic. Although some peripheral regions have an opportunistic mentality of "freeriding" on the governance in other regions, resulting in an evolutionary game that does not lead to joint regional governance, it is still the most efficient outcome for some regions to choose nongovernance as long as the spillover from regional governance to other regions is large enough.

(2) In the game of governance between the core and peripheral regions of a public health outbreak, governance of the public health outbreak is an equilibrium stabilization strategy for the core region. Besides, whether the peripheral region is governed or not depends on the spillover effect of the governance of the core region on the peripheral region, the cost of governance in the peripheral region and the losses incurred from the public health outbreak. When the spillover effect is large, the cost of peripheral area governance is small, and the loss from public health outbreak is small, peripheral areas have the "freerider" mentality and tend to choose nongovernance. In this case, joint governance between core areas and peripheral areas cannot be achieved, and public health emergencies cannot be effectively governed. For the current management of the new crown 
epidemic, the core regions of the outbreak choose to manage the epidemic due to the great harm it causes, but the spillover effect of its management on some peripheral regions is small. Thus, if the peripheral regions do not choose strict management and control measures, there will be a greater risk of outbreaks. As a result, they will also engage in the management of the outbreaks by cooperating with the core regions, while few peripheral regions of the core choose not to do so.

(3) To suppress the inefficient "free-riding" phenomenon in the cross-regional governance game of public health emergencies, the benefits of regional "freeriding" are changed by constructing and controlling a cross-regional management platform and providing rewards and penalties for nongovernance areas. When the reward and punishment for nongovernance areas are greater than a certain threshold, all inefficient "free-riding" will disappear, and the equilibrium of the regional governance game is the socially optimal governance. Therefore, for the current management of the new crown epidemic, in order to ensure effective multiregional joint management, a multiregional joint management platform can be established for coordination and control to achieve effective multiregional collaborative management through rewards and penalties.

Based on the above findings, and to improve the effectiveness of cross-regional governance of public health emergencies, this paper makes the following recommendations.

First, a regional joint governance platform should be established to provide unified coordination of regional public health governance. The government should also establish a cross-regional joint governance system for public health incidents, strengthen regional cooperation and exchange, study and develop cross-regional collaborative and joint working mechanisms, provide unified coordination and management of multiregional governance, and supervise and punish areas that do not take relevant governance measures. Furthermore, it is advisable to promote joint missions among cross-regional emergency management departments, to establish cross-regional and cross-sectoral communication and coordination networks for communication, and to encourage cross-regional cooperation among public health governance-related departments.

Second, while there is regional "free-riding" in the governance of public health among regions with large spillover effects, not all "free-riding" affects the governance of public health events in society as a whole. In this case, cross-regional management platforms should not overreact and intervene.

Third, the big data platform should be leveraged to share information on public health events across regions, and a cross-regional public health event platform for monitoring, assessment, and early warning should be established to achieve precise governance of public health emergencies and reduce regional governance costs. Additionally, we need to strengthen real-time information sharing and seamless integration on cross-regional public health events and share typical experiences and practices. The regions involved should be unified in the deployment of rescue forces and material resources and an intercity and interregional realtime big data platform should be built to share information on the epidemic. Finally, a cross-city and cross-regional cooperation risk governance body should be established, and infrastructure should be co-constructed and shared across administrative boundaries.

Finally, in this paper, we use evolutionary game method to study the cross-border governance decision of public health events, assuming that the regional governance decision set is governance or nongovernance, but in the actual regional decision-making, the set of its choice includes the intermediate state of governance and nongovernance. The regional governance decision may be fuzzy; while Pythagorean is an important tool to solve the uncertainty problem in multiattribute decision-making [27-29], it will be the direction of further research in this paper to study the crossborder governance of public health events by integrating Pythagorean fuzzy decision-making method with evolutionary game method.

\section{Data Availability}

The data used to support the findings of this study are included within the article.

\section{Conflicts of Interest}

The authors declare that there are no conflicts of interest regarding the publication of this paper.

\section{Acknowledgments}

This paper was supported by the Institute of National Accounts (Zhuhai), Beijing Normal University. This research was funded by the National Natural Science Foundation of China (Grant no. 71873019) and the National Social Science Fund of China (Grant no. 18ZDA123).

\section{References}

[1] S. Zhao, S. S. Musa, Q. Lin et al., "Estimating the unreported number of novel coronavirus (2019-nCoV) cases in China in the first half of january 2020: a data-driven modelling analysis of the early outbreak," Journal of Clinical Medicine, vol. 9, no. 2, p. 388, 2020.

[2] J. She, J. Jiang, L. Ye et al., "Novel coronavirus of pneumonia in Wuhan, China: emerging attack and management strategies," Clinical and Translational Medicine, vol. 9, no. 1, pp. 1-7, 2019.

[3] Q. Ding, P. Lu, Y. Fan et al., "The clinical characteristics of pneumonia patients coinfected with 2019 novel coronavirus and influenza virus in Wuhan, China," Journal of Medical Virology, vol. 92, no. 9, pp. 1-7, 2020.

[4] M.-C. Besner, M. Prévost, and S. Regli, "Assessing the public health risk of microbial intrusion events in distribution systems: conceptual model, available data, and challenges," Water Research, vol. 45, no. 3, pp. 961-979, 2011. 
[5] H. Tu, S. Tu, S. Gao, et al., The epidemiological and clinical features of COVID-19 and lessons from this global infectious public health event," Journal of Infection, vol. 81, no. 1, pp. 1-9, 2020.

[6] E. Fearnley and L. Ailan, "Public health event communications in the western pacific region," western pacific surveillance \& response," Journal Wpsar, vol. 4, no. 3, p. 26, 2005.

[7] R. J. Wray, S. M. Becker, N. Henderson et al., "Communicating with the public about emerging health threats: lessons from the pre-event message development project," American Journal of Public Health, vol. 98, no. 12, pp. 2214-2222, 2008.

[8] J. M. Smith and G. R. Price, "The logic of animal conflict," Nature, vol. 246, no. 5427, pp. 15-18, 1973.

[9] M.. Smith, Evolution and the Theory of Games, Cambridge University Press, Cambridge, UK, 1982.

[10] J. Hofoauer and K. Siglnund, "Evolutionary game dynamies," Bulletin-Ameriean Mathematieal Soeiety, vol. 40, no. 4, pp. 479-520, 2003.

[11] B. J. Harvey and L. C. Loh, "Public health medicine, public health practice, and public health systems authors' response," Canadian Journal of Public Health, vol. 104, no. 2, pp. 117127, 2013.

[12] R. Philipp, N. Gibbons, P. Thorne, L. Wiltshire, J. Burrough, and J. Easterby, "Evaluation of a community arts installation event in support of public health," Perspectives In Public Health, vol. 135, no. 1, pp. 43-48, 2015.

[13] C. Stevinson and M. Hickson, "Exploring the public health potential of a mass community participation event," Journal of Public Health, vol. 36, no. 2, pp. 268-274, 2014.

[14] B. Sahoh, "Smart emergency management based on social big data analytics: research trends and future directions," in Proceedings of the 2017 International Conference, pp. 1-6, New York, NY, USA, 2017.

[15] J. Beltrami and S. Berman, "Congenital syphilis: a persisting sentinel public health event," Sexually Transmitted Diseases, vol. 33, no. 11, pp. 675-676, 2006.

[16] A. Kolbe and K. L. Gilchrist, "An extreme bushfire smoke pollution event: health impacts and public health challenges," New South Wales Public Health Bulletin, vol. 20, no. 1, pp. 19-20, 2009.

[17] L. S. Meredith, D. P. Eisenman, H. Rhodes et al., "Trust influences response to public health messages during a bioterrorist event," Journal of Health Communication, vol. 12, no. 3, pp. 217-232, 2007.

[18] S. Akter and S. F. Wamba, "Big data and disaster management: a systematic review and agenda for future research," Annals of Operations Research, vol. 283, no. 1, pp. 939-959, 2019.

[19] Y.. Wang, J.. Shen, and Z.. Jiang, "Analysis of the game influence of information behavior on decision makers in public health emergencies: case study of COVID-19 event," Information Research, vol. 7, pp. 57-63, 2020.

[20] Yu. Lei and H. F. Xue, "Simulation of emergency management system of public health based on evolutionary game," Computer Simulation, vol. 25, no. 4, pp. 169-171, 2008.

[21] D. Liu, J. Chen, R. Chai et al., "Evolutionary analysis of important public health event-based multi-agent simulation model," International Journal of Innovative Computing \& Applications, vol. 6, no. 1, 2014.

[22] D. D. Luxton, J. D. June, and J. M. Fairall, "Social media and suicide: a public health perspective," American Journal of Public Health, vol. 102, no. 2, pp. 195-201, 2012.
[23] M. Fisichella, A. Stewart, K. Denecke et al., "Unsupervised public health event detection for epidemic intelligence," ACM, vol. 1, no. 4, pp. 1881-1884, 2010.

[24] Y.. Xiao, Q.. Peng, W.. Xu, and H.. Xiao, "Production-use water pricing and corporate water use in China: an evolutionary game theory model," Mathematical Problems in Engineering, vol. 1, no. 3, pp. 1-9, 2021.

[25] Q.. Peng and Y.. Xiao, "Will third-party treatment effectively solve issues related to industrial pollution in China?" Sustainability, vol. 12, no. 18, p. 7685, 2020.

[26] Y.. Yang and W.. Yang, "Does whistleblowing work for air pollution control in China? A study based on three-party evolutionary game model under incomplete information," Sustainability, vol. 11, no. 2, pp. 324-344, 2019.

[27] S. Z. Zeng, Y. J. Hu, T. Balezentis, and D. Streimikiene, "A multi-criteria sustainable supplier selection framework based on neutrosophic fuzzy data and entropy weighting," Sustainable Development, vol. 28, no. 5, pp. 1431-1440, 2020.

[28] C. H. Zhang, W. H. Su, S. Z. Zeng, T. Balezentis, and E. Herrera-Viedma, "A two-stage subgroup decision-making method for processing large-scale information," Expert Systems with Applications, vol. 171, no. 3, p. 114586, 2021.

[29] Z. M. Mu, S. Z. Zeng, and P. Y. Wang, "Novel approach to multi-attribute group decision-making based on intervalvalued Pythagorean fuzzy power Maclaurin symmetric mean operator," Computers \& Industrial Engineering, vol. 155, p. 107049, 2021. 